\title{
Analysis on the Suitability of Residential Buildings from the Perspective of Systematic Integration
}

\author{
Lei Chen ${ }^{1}$ \\ ${ }^{1}$ University of South China, Hunan 421001, China;
}

Keywords: Systematic Integration, Suitability, Residential Buildings, Perspective.

\begin{abstract}
This paper proposes the idea of suitability of residential buildings from the perspective of the systematic integration. Architect's responsibility is in each kind of the material foundation decided which new function system can advocate, and that causes the people maximum limit benefit in the construction uses the metaphor technique to be possible to help the architect to achieve this goal. Construction objective environment there are differences between east and west, the difference is not due to the convenience of the modern social information transmission become about change or disappears that architectural works. Guided by this basic perspective, this paper proposes the novel paradigm for the suitability of residential buildings. The proposed method will help us to finalize the future developmental orientation of the modern architecture.
\end{abstract}

\section{Introduction}

Modern science and technology revolution, after is continues in the modern history first, two section of science and technology revolution third science and technology revolution, it has become one of present era basic characteristics, not only the influence social economy, political, and the cultural development, caused has thrown over the huge change which and the global humanity lived that will restrict the human history the future, and unfolded to the contemporary society proposed a series of the new topics. Buildings has its inner meaning, has a practical functional and symbolic. Significance of the building is a very important aspect, and that often neglected in the past, the meaning of the construction must be through the contact and perception to be understood.

Building, of course, not only for the purpose of the communication, it is more to create the better environment for human beings. Metaphor is using the perspective of semiotics, the building as a language and its basic carrier is common and natural language, by practical, the structural reliability factors, economy and technology equipment, etc. Construction of the poetic language is embodied in appearance, the space form, social customs and etiquette, etc.

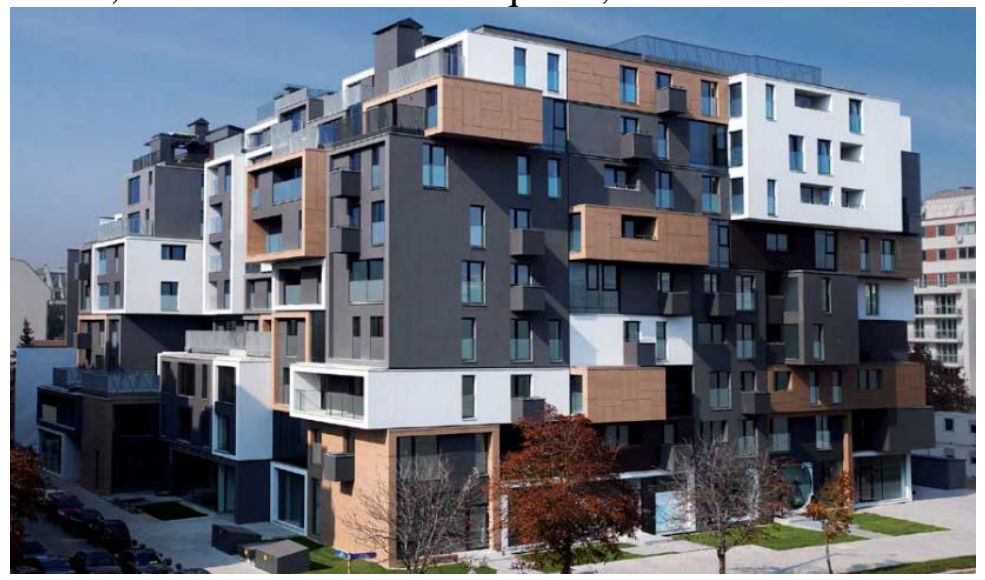

Fig. 1 Residential Buildings Illustration

As shown in the figure one, we illustrate the residential buildings. With continuous development of intelligent systems, intelligent systems shall deposit with the energy saving, green building symbiosis, the function of the intelligent system, performance and operation will be an integral part of the green building, green building to realize most of the target and implementation scheme is inseparable from the intelligent system. Under this basis, in the later sections, we will discuss in detail. 


\section{Our Proposed Methodology}

System Integration Thinking. From the different Angle analysis, information system integration has a different explanation. Meaning is the essence of system integration was structured integrated wiring system and computer network technology will the separation of the data devices (such as personal computers), function and information integration to the interrelated, unity and coordination system, make to achieve full sharing of resources, concentrated, efficient and convenient management.

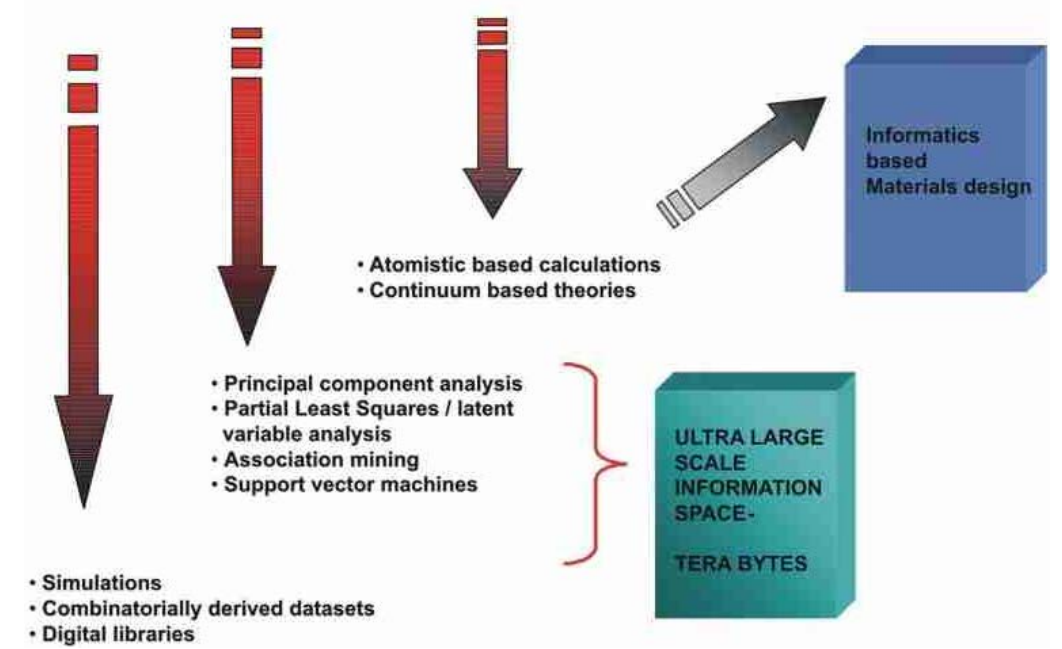

Fig. 2 System Integration Thinking Organizations and Procedures

Therefore, the information system has the following core characteristics: (1) information system integration to meet the needs of users as the starting point; (2) information system integration is not a simple choice of the best products of the general behavior, but to choose the most suitable for user needs and investment scale of the product and technology; (3) information system integration is not a simple equipment supply it then embodies more general planning, optimization, organization, design, commissioning and development as is a high technical content of integrated behavior; (4) information system integration includes technology, Management and business, etc., is a comprehensive system engineering, technology is the core of modern information systems integration work, management and business activities is the information system integration project successfully implemented the reliable guarantee; (5) information system integration should consider the cost and efficiency factors, The level of performance and price ratio is an important reference factor in evaluating whether an information system integration project design is reasonable and successful.

The data integration is a basic integration and the method mainly has the data format definition, the rule description, the data reorganization and the retreatment. The data integration needs to solve the different data pool consistent view problem that carries on the different database system content the whole the organization. The data must be able easy to inquire, and can uniquely mark in the entire integrated application scope. In the various objects of the information system integration, network integration is the hard foundation of the information system integration, and data as the carrier of information resources, is the soft foundation of information system integration, but also the primary task of information system integration. Data integration for information systems to provide the basis for integration, build platform while no data integration, the various internal information is scattered, messy "information island" that cannot provide the valuable information and knowledge management decision-making that cannot be used for enterprises.

Modern Architecture Review and Analysis. Metaphorism's implication of general historical architecture and traditional culture can enrich new building content and make people more familiar with new buildings. Therefore, the more the more complex the metaphor of the more visual impact and artistic appeal, it can meet the different levels of the crowd's sensory needs. And modernism has never looked up the classical vocabulary and the meaning of local dialects, that the reference to the traditional or symbol to symbolic is not creative, it is not enough, which also makes a lot of modernist 
architecture has become thin and lack of heritage as after the entire modernism, the construction and populace's relations rose a very high boundary. The metaphor principle on pays great attention to the construction and populace's exchange extremely, but creates one kind diligently both newly and the construction which echoes with the basic letter code. The construction language has the psychological instruction function, it can use in one form processing to direct the populace, enables it to understand the construction information gives the suggestion, suggested some kind of recent trend of development, including aspects and so on history, culture, technological development, suggested how user's demand should change. The contemporary west thought generally the architect must use the mass culture form the building must conform to populace's requirement.

Architect's responsibility is in each kind of the material foundation decided which new function system can advocate, and that causes the people maximum limit benefit in the construction uses the metaphor technique to be possible to help the architect to achieve this goal. Construction objective environment there are differences between east and west, the difference is not due to the convenience of modern social information transmission become about change or disappear that architectural works should be rooted in tradition and nature kingdom of a core nation works difference is very obvious architectural creation view is: don't let age before then ignore old civilization: that tries to learn the traditional essence, characteristic construction is also endowed with some of new era. Architecture development today, people's attention has shifted away from the building itself increasingly turned to related to the construction environment, buildings and the environment it is space of a whole system, it is to sustain human production, life and cultural activities in various fields such as dynamic balance between elements that can be analyzed and understood from the listed perspectives.

- Building image and objective vivid and the image of the body, even, the fusion of different subject for the same image building has a different feelings' will be saturated with subject such as the aesthetic meaning.

- Architectural imagery has the physical image and the spatial image of the multiplicity, because the human participation in the building space image has static image and dynamic image, the building entity image has two-dimensional image and some of the three-dimensional image characteristics, it is multi-level environmental image, the multiplicity of the image makes it a composite aesthetic artistic feature.

- Art to show the real life and appeal to the highest state of nature, as a kind of art, building with presence of artistic conception 'artistic conception of the degree of depth and determine the style of the artistic conception is the soul of artistic creation, and architectural aesthetics in the process of the subjects' to the creation of the architectural image just as artistic conception creation of intermediaries' building image means rather than aim is to create realm.

Building Assessment. From around the world experience, green building generally belongs in the realm of social public welfare is stronger, and the market mechanism failure, just rely on market mechanism is difficult to make up its rapid development, only through policy fully by means of legal, administrative, and economic incentives, to guide, standardize and promote the development of the work, such as early in promoting the development of intelligent integration of the green building, the government needs to take a series of subsidies, tax and other measures. For the general analysis for the assessment of the building, we propose the following suggestions.

- Try to minimize the use of wood in residential construction. Using standard components to reduce the use of wooden template synthesis as the use of solid, after using the template, such as steel template instead of wood template packed in strong, repeated use, instead of wooden packaging.

- Residential requirements of the plane can be flexible changes, the installation of the column beam to facilitate the separation of space. Note that the $6 \mathrm{~m}$ large space separation, the use of lightweight partitions, pipes and pipelines easy to modify, modify the layout and decoration will produce a large number of construction waste, Interior wall materials and bathroom and he kitchen facilities are required to be reused for easy replacement. 
- Indoor public areas, and such as: the elevator lobby, staircase, equipment, etc., to set up the necessary lighting. Outdoor activities, such as playground, sidewalks, service place, also want to combine, set up necessary lighting. The lighting involved to consider energy use, lamps and lanterns of lighting levels, and energy-saving control.

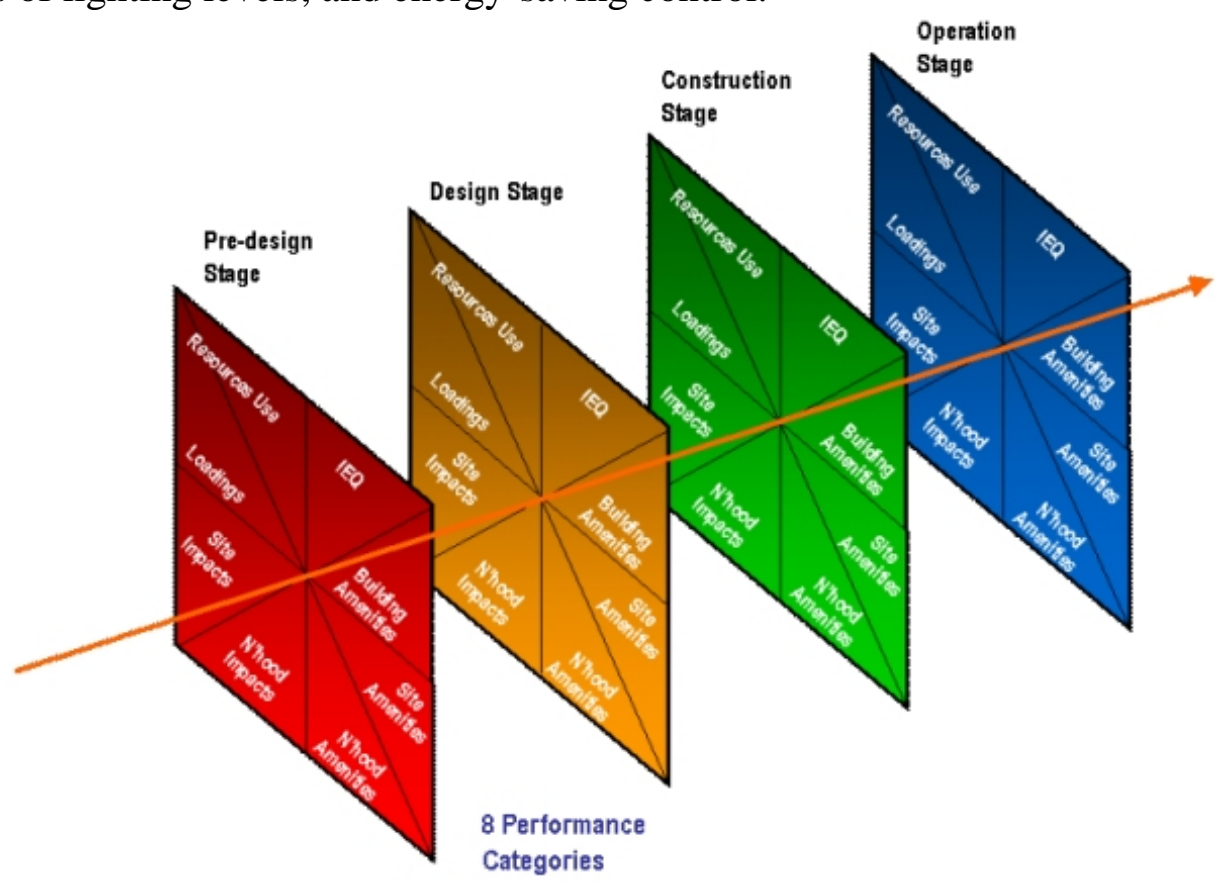

Fig. 2 Building Assessment Stages

\section{Summary and Conclusion}

Building for the "people", people-oriented building is the key to the development. Intelligent green building is different from the traditional building, and its construction concept across the building ontology and the pursuit of modern human survival goals optimization, the fundamental goal is in the construction of the "life" cycle, to maximize the protection of the environment, saving resources (energy, Water, land, and materials) and reduce pollution, to provide people with healthy, applicable and efficient use of space, and ultimately achieve symbiosis with nature, to achieve the sustainable development of cities and buildings. Under this basis, this paper proposes the idea of suitability of residential buildings from the perspective of systematic integration. In the future, we will later use more integration to enhance the current research.

\section{References}

[1] Samuel, Laura J., Yvonne Commodore-Mensah, and Cheryl R. Dennison Himmelfarb. "Developing behavioral theory with the systematic integration of community social capital concepts." Health Education \& Behavior 41.4 (2014): 359-375.

[2] Martinie, Célia, et al. "Extending procedural task models by explicit and systematic integration of objects, knowledge and information." (2013): 1-10.

[3] Sanborn, Graham, et al. "Systematic Integration of Finite Element Methods Into Multibody Dynamics Considering Hyperelasticity and Plasticity." Journal of Computational and Nonlinear Dynamics 9.4 (2014): 041012.

[4] Landherr, Martin, and Engelbert Westkämper. "Integrated product and assembly configuration using systematic modularization and flexible integration." Procedia CIRP 17 (2014): 260-265. 